\title{
Schwinger effect from near-extremal black holes in (A)dS space
}

\author{
Chiang-Mei Chen๑* \\ Department of Physics, National Central University, Chungli 32001, Taiwan \\ and Center for High Energy and High Field Physics (CHiP), National Central University, \\ Chungli 32001, Taiwan \\ Sang Pyo Kim ${ }^{\dagger}$ \\ Department of Physics, Kunsan National University, Kunsan 54150, Korea \\ and Institute of Theoretical Physics, Chinese Academy of Sciences, Beijing 100190, China
}

(Received 10 February 2020; accepted 2 April 2020; published 24 April 2020)

\begin{abstract}
We study the Schwinger effect in near-extremal Reissner-Nordström black holes with electric and/or magnetic charges in (anti-)de Sitter (AdS) space. The formula for the Schwinger effect takes a universal form for near-extremal black holes with the near-horizon geometry of $\mathrm{AdS}_{2} \times S^{2}$ and with the proper radii for the $\mathrm{AdS}_{2}$ space and the two-sphere $S^{2}$, regardless of the asymptotically flat or (A)dS space. The asymptotic AdS boundary enhances and the dS boundary suppresses the Schwinger effect, and the small radius of AdS (dS) space reinforces the enhancement and suppression.
\end{abstract}

DOI: 10.1103/PhysRevD.101.085014

\section{INTRODUCTION}

Quantum fluctuations spontaneously create particle pairs from the vacuum unless there exists an external mechanism preventing annihilation of pairs. The event horizon of a black hole casually separates pairs into the interior and the exterior, and the black hole emits all species of particles [1]. A sufficiently strong electric field separates charged pairs and accelerates them in opposite directions, the so-called Schwinger pair production [2,3]. The Hawking radiation and Schwinger effect are consequences of the nonperturbative effects of quantum field theory.

The Hawking radiation and Schwinger effect both act as the emission channels of charged pairs from charged black holes. Hawking radiation obeying the Bose-Einstein or Fermi-Dirac distribution with the Hawking temperature emits charges with a Coulomb chemical potential on the horizon. Though the Hawking radiation from (near-) extremal black holes is exponentially suppressed due to vanishing Hawking temperature, the Schwinger mechanism triggers the emission of charges. The Schwinger effect has been extensively studied for (near-)extremal ReissnerNordström (RN) black holes [4,5], Kerr-Newman (KN)

\footnotetext{
cmchen@phy.ncu.edu.tw

†sangkim@kunsan.ac.kr
}

Published by the American Physical Society under the terms of the Creative Commons Attribution 4.0 International license. Further distribution of this work must maintain attribution to the author(s) and the published article's title, journal citation, and DOI. Funded by SCOAP ${ }^{3}$. black holes [6], and dyonic KN black holes [7] (for review and references, see Ref. [8]). The Schwinger fermion production has also been studied for charged black holes in $(\mathrm{A}) \mathrm{dS}$ space [9-11].

Primordial black holes have been studied in cosmology and astrophysics [12-14]. It was argued that dynonic extremal black holes are stable against Hawking radiation [15]. Black holes of Planck scale are stable quantum mechanically and can be candidates for dark matter [16]. The Schwinger effect from (near-)extremal RN and dyonic RN black holes in (anti-)de Sitter space is interesting theoretically and cosmologically since the asymptotic (A)dS boundary affects the near-horizon geometry of (near-)extremal black holes [17]. In particular, the expanding early universe described by $\mathrm{dS}$ space drastically changes the property and evolution of charged black holes.

We study the effect of the asymptotic (A)dS boundary on Schwinger pair production in (near-)extremal RN black holes. The extremal RN-(A)dS black holes are obtained by degenerating the event horizon and the causal horizon while keeping the cosmological horizon separate from the black hole horizon. The near-horizon region of (near-) extremal RN-(A)dS black holes has the geometry of $\mathrm{AdS}_{2} \times S^{2}$, whose symmetry allows explicit solutions of a charged field in terms of confluent hypergeometric function. The asymptotic (A)dS boundary makes the effective radii of the $\mathrm{AdS}_{2}$ and the two-sphere being different from that of (near-) extremal RN black holes in the asymptotically at space [4,5]. The formula for the mean number for pair production has a universal structure in terms of the effective temperature for charge 
acceleration and Hawking temperature with modifications due to the effect of the asymptotic (A)dS boundary. We then investigate the pair production of dyons with electric and magnetic charges from (near-)extremal dyonic RN-(A)dS black holes. The formula for the mean number of dyon pairs exhibits the universal structure.

The organization of this paper is as follows. In Sec. II, we study the Schwinger effect in (near-)extremal RN-(A)dS black holes. The near-horizon geometry is still $\mathrm{AdS}_{2} \times S^{2}$, whose effective radii for $\mathrm{AdS}_{2}$ space and $S^{2}$ are modified by the asymptotic radius of (A)dS space. The Schwinger effect has a universal structure in terms of the effective temperature for charges and the Hawking temperature, and is suppressed by the dS boundary but enhanced by the AdS boundary. In Sec. III, the Schwinger effect is studied in (near-)extremal dyonic black holes in (A)dS space. The universal structure for the mean number of dyon pairs shows the Schwinger effect in $\mathrm{AdS}_{2}$ and the quantum electrodynamics (QED) effect of the electromagnetic field in Rindler space for the surface gravity of the event horizon. The effective temperature is determined by the Unruh temperature for the acceleration of dyons in the electromagnetic field. The Schwinger effect is suppressed in dS space but enhanced in AdS space. In Sec. IV, we discuss cosmological and astrophysical implications of the effect of the asymptotic (A)dS boundary on the Schwinger effect.

\section{II. (NEAR-)EXTREMAL RN-(A)dS BLACK HOLES}

The action for RN-(A)dS black holes is given by

$$
S=\int d^{4} x \sqrt{-g}\left(R \pm \frac{6}{L^{2}}-F_{\mu \nu} F^{\mu \nu}\right),
$$

where a cosmological constant $\Lambda=\mp 3 / L^{2}$ (the upper/ lower sign) here and hereafter corresponds to AdS/dS space, respectively. The metric for a $\mathrm{RN}-(\mathrm{A}) \mathrm{dS}$ black hole is

$$
\begin{aligned}
d s^{2} & =-f(r) d t^{2}+\frac{d r^{2}}{f(r)}+r^{2} d \Omega_{2}^{2}, \\
f(r) & =1-\frac{2 M}{r}+\frac{Q^{2}}{r^{2}} \pm \frac{r^{2}}{L^{2}}, \\
A & =\frac{Q}{r} d t,
\end{aligned}
$$

where $M$ and $Q$ are the mass and charge of the black hole and $L$ is the (A)dS radius. There are two positive roots of $f(r)$-in general, associated with the causal horizon $r_{-}$and event horizon $r_{+}$-and for the $\mathrm{dS}$ case a third positive root corresponds to the cosmological horizon $r_{\mathrm{C}}$ [18]. Out of three horizons of $\mathrm{RN}-(\mathrm{A}) \mathrm{ds}$ black holes (2), the causal horizon $r_{-}$and the event horizon $r_{+}$are made degenerate, $M=M_{0}, r_{+}=r_{-}=r_{0}$, to yield an extremal RN-(A)dS black hole, where

$$
\begin{aligned}
M_{0} & =\frac{L(3 \pm \delta) \sqrt{\delta}}{3 \sqrt{6}}, \quad r_{0}=\frac{L \sqrt{\delta}}{\sqrt{6}}, \\
\delta & = \pm\left(\sqrt{1 \pm 12 Q^{2} / L^{2}}-1\right) .
\end{aligned}
$$

The cosmological horizon remains outside of the event horizon $\left(r_{\mathrm{C}}>r_{0}\right)$. By elongating the radial coordinate $r=r_{0}+\epsilon \rho$ and reducing the time coordinate, $t=\tau / \epsilon$, one has the near-horizon geometry of extremal RN-(A)dS,

$d s^{2}=-\frac{\rho^{2}}{R_{\mathrm{AdS}}^{2}} d \tau^{2}+\frac{R_{\mathrm{AdS}}^{2}}{\rho^{2}} d \rho^{2}+R_{\mathrm{S}}^{2} d \Omega_{2}^{2}, \quad A=-\frac{Q}{R_{\mathrm{S}}^{2}} \rho d \tau$,

possessing a structure of $\mathrm{AdS}_{2} \times S^{2}$ with radii

$$
R_{\mathrm{AdS}}^{2}=\frac{L^{2} \delta}{6(1 \pm \delta)}, \quad R_{\mathrm{S}}^{2}=r_{0}^{2}=\frac{L^{2} \delta}{6} .
$$

From Eq. (3), we find another expression

$$
\begin{aligned}
R_{\mathrm{S}}= & \frac{L}{\sqrt{6}}\left[\left(\sqrt{1+\frac{27}{2} \frac{M_{0}^{2}}{L^{2}}}+\sqrt{\frac{27}{2}} \frac{M_{0}}{L}\right)^{1 / 3}\right. \\
& -\left(\sqrt{1+\frac{27}{2} \frac{M_{0}^{2}}{L^{2}}}+\sqrt{\left.\left.\frac{27}{2} \frac{M_{0}}{L}\right)^{-1 / 3}\right]},\right.
\end{aligned}
$$

for AdS space, and

$$
\begin{aligned}
R_{\mathrm{S}}= & -i \frac{L}{\sqrt{6}}\left[\left(\sqrt{1-\frac{27}{2} \frac{M_{0}^{2}}{L^{2}}}+i \sqrt{\frac{27}{2} \frac{M_{0}}{L}}\right)^{1 / 3}\right. \\
& \left.-\left(\sqrt{1-\frac{27}{2} \frac{M_{0}^{2}}{L^{2}}}+i \sqrt{\frac{27}{2}} \frac{M_{0}}{L}\right)^{-1 / 3}\right]=\frac{2 L}{\sqrt{6}} \sin \frac{\vartheta}{3}, \\
\vartheta= & \sin ^{-1} \sqrt{\frac{27}{2}} \frac{M_{0}}{L}, \quad 0 \leq \vartheta \leq \pi / 2,
\end{aligned}
$$

for dS space. The black hole radius takes the large $L$ limit

$$
\begin{aligned}
R_{\mathrm{S}}= & M_{0}\left[1 \mp 2\left(\frac{M_{0}}{L}\right)^{2}+12\left(\frac{M_{0}}{L}\right)^{4} \mp 96\left(\frac{M_{0}}{L}\right)^{6}\right. \\
& \left.+\mathcal{O}\left(\frac{M_{0}}{L}\right)^{8}\right] .
\end{aligned}
$$

The radius of $\mathrm{AdS}_{2}$ and the charge are

$$
R_{\text {AdS }}^{2}=\frac{R_{\mathrm{S}}^{2}}{1 \pm 6\left(\frac{R_{\mathrm{S}}}{L}\right)^{2}}, \quad Q=R_{\mathrm{S}} \sqrt{1 \pm 3\left(\frac{R_{\mathrm{S}}}{L}\right)^{2}} .
$$

We consider a charged scalar governed by the KleinGordon equation, 


$$
\left(\nabla_{\mu}-i q A_{\mu}\right)\left(\nabla^{\mu}-i q A^{\mu}\right) \Phi-m^{2} \Phi=0,
$$

whose solution

$$
\Phi(\tau, \rho, \theta, \varphi)=\mathrm{e}^{-i \omega \tau+i n \varphi} R(\rho) S(\theta)
$$

is separated into the angular and radial equations as

$$
\begin{gathered}
\frac{1}{\sin \theta} \partial_{\theta}\left(\sin \theta \partial_{\theta} S\right)-\left(\frac{n^{2}}{\sin ^{2} \theta}-l(l+1)\right) S=0, \\
\partial_{\rho}\left(\rho^{2} \partial_{\rho} R\right)+\left[\frac{R_{\mathrm{AdS}}^{4}}{R_{\mathrm{S}}^{4}} \frac{\left(q Q \rho-\omega R_{\mathrm{S}}^{2}\right)^{2}}{\rho^{2}}-R_{\mathrm{AdS}}^{2} m^{2}\right. \\
\left.-\frac{R_{\mathrm{AdS}}^{2}}{R_{\mathrm{S}}^{2}} l(l+1)\right] R=0 .
\end{gathered}
$$

By introducing an effective mass and $R_{\mathrm{AdS}}^{2}$-rescaled electric force

$$
\bar{m}^{2}=m^{2}+\frac{l(l+1)}{R_{\mathrm{S}}^{2}}+\frac{1 / 4}{R_{\mathrm{AdS}}^{2}}, \quad \kappa=R_{\text {AdS }}^{2} \frac{q Q}{R_{\mathrm{S}}^{2}},
$$

the violation of the Breitenlohler-Freedman (BF) bound in $\mathrm{AdS}_{2}$ space leading to the Schwinger pair production takes the form [19-21]

$$
\mu^{2}=\kappa^{2}-R_{\text {AdS }}^{2} \bar{m}^{2} \geq 0 .
$$

The BF bound $\left(\mu^{2}<0\right)$ of $\mathrm{AdS}_{2}$ space guarantees the stability against the pair production. The solution to the radial equation is in terms of the Whittaker function [4]

$$
R=c_{1} M_{i \kappa, i \mu}(z)+c_{2} M_{i \kappa,-i \mu}(z), \quad z=i \frac{2 \omega R_{\mathrm{AdS}}^{2}}{\rho} .
$$

According to our earlier result in Ref. [4], the mean number of produced pairs is

$$
\begin{aligned}
\mathcal{N} & =\frac{\sinh (2 \pi \mu)}{\cosh (\pi \kappa+\pi \mu)} \exp (\pi \mu-\pi \kappa) \\
& =\frac{\mathrm{e}^{-2 \pi(\kappa-\mu)}-\mathrm{e}^{-2 \pi(\kappa+\mu)}}{1+\mathrm{e}^{-2 \pi(\kappa+\mu)}}
\end{aligned}
$$

The mean number (17) of charges from the extremal black hole has a thermal interpretation

$$
\mathcal{N}=\frac{\mathrm{e}^{-\bar{m} / T_{\mathrm{S}}}-\mathrm{e}^{-\bar{m} / \bar{T}_{\mathrm{S}}}}{1+\mathrm{e}^{-\bar{m} / \bar{T}_{\mathrm{S}}}},
$$

in terms of the effective temperature
$T_{\mathrm{S}}=\frac{\bar{m}}{2 \pi(\kappa-\mu)}=T_{\mathrm{U}}+\sqrt{T_{\mathrm{U}}^{2}-\frac{1}{4 \pi^{2} R_{\mathrm{AdS}}^{2}}}, \quad T_{\mathrm{U}}=\frac{\kappa / R_{\mathrm{AdS}}^{2}}{2 \pi \bar{m}}$,

$\bar{T}_{\mathrm{S}}=\frac{\bar{m}}{2 \pi(\kappa+\mu)}=T_{\mathrm{U}}-\sqrt{T_{\mathrm{U}}^{2}-\frac{1}{4 \pi^{2} R_{\mathrm{AdS}}^{2}}}$.

Note that the effective temperature (19) is the same as that of charges in a uniform electric field in $\mathrm{AdS}_{2}$ space, in which $T_{\mathrm{U}}$ is the Unruh temperature for the accelerated charge by the electric field, and the second term in the square root is related to the $\mathrm{BF}$ bound $[19,20]$, which corresponds to the Gibbons-Hawking temperature squared in $\mathrm{dS}_{2}$ space [21]. The factor of 2, i.e., $T_{\mathrm{S}}=2 T_{\mathrm{U}}$, in the Minkowski spacetime limit $\left(R_{\mathrm{AdS}}=\infty\right)$, is an ultrarelativistic feature of the Schwinger effect. In general, $T_{\mathrm{S}}$ for AdS space is larger, and $T_{\mathrm{S}}$ for $\mathrm{dS}$ space is smaller than that for the asymptotically flat space, as shown in Fig. 1 . The $T_{\mathrm{S}}$ for (A)dS space approaches that for asymptotically flat space. The reason for this is that the $\mathrm{AdS}_{2}$ radius $R_{\mathrm{AdS}}$ and the two-sphere radius $R_{\mathrm{S}}$ for $\mathrm{dS}$ space are larger than those for AdS space (see Fig. 1); thereby, the electric field on the horizon is weaker, and the Unruh temperature lower for $\mathrm{dS}$ space than those for AdS space. The asymptotic (A)dS space drastically changes the effective temperature: the higher temperature for AdS space enhances the Schwinger effect, while the lower temperature for dS space suppresses the Schwinger effect. Remarkably, dS space increases the $\mathrm{AdS}_{2}$ radius $R_{\mathrm{AdS}}$ for the $\mathrm{BF}$ bound, which implies larger extremal RN black holes that are stable against the Schwinger effect compared to asymptotically flat space; this opens the possibility for large extremal primordial black holes.

The dependence of $T_{\mathrm{S}}$ and radii $R_{\mathrm{S}}, R_{\text {AdS }}$ with respect to $M_{0}$ is given in Fig. 2. The effect of the cosmological constant becomes more significant, enhanced in AdS and suppressed in $\mathrm{dS}$, when $M_{0}$ is larger. The small $M_{0}$ threshold in $T_{\mathrm{S}}$ reflects the $\mathrm{BF}$ bound in (15). The Schwinger effect is actually in accordance with the unstable modes of $\mathrm{AdS}_{2}$ in near-horizon geometry. For the asymptotically dS spacetimes, there is an upper bound of $M_{0}$ such that the Schwinger mechanism is terminated. This corresponds to the limit when the black hole horizon $R_{\mathrm{S}}$ approaches the cosmological horizon, namely $\delta=1$ or $\vartheta=\pi / 2$ in (7), and then the $R_{\text {Ads }}$ diverges.

The near-horizon geometry of near-extremal RN black holes is obtained by scaling coordinates $r_{ \pm}=r_{0} \pm \epsilon B$ and parametrizing the mass

$$
M=M_{0}+(\epsilon B)^{2} \frac{R_{\mathrm{S}}}{2 R_{\mathrm{AdS}}^{2}}
$$

The metric and gauge fields for the near-extremal black holes are 

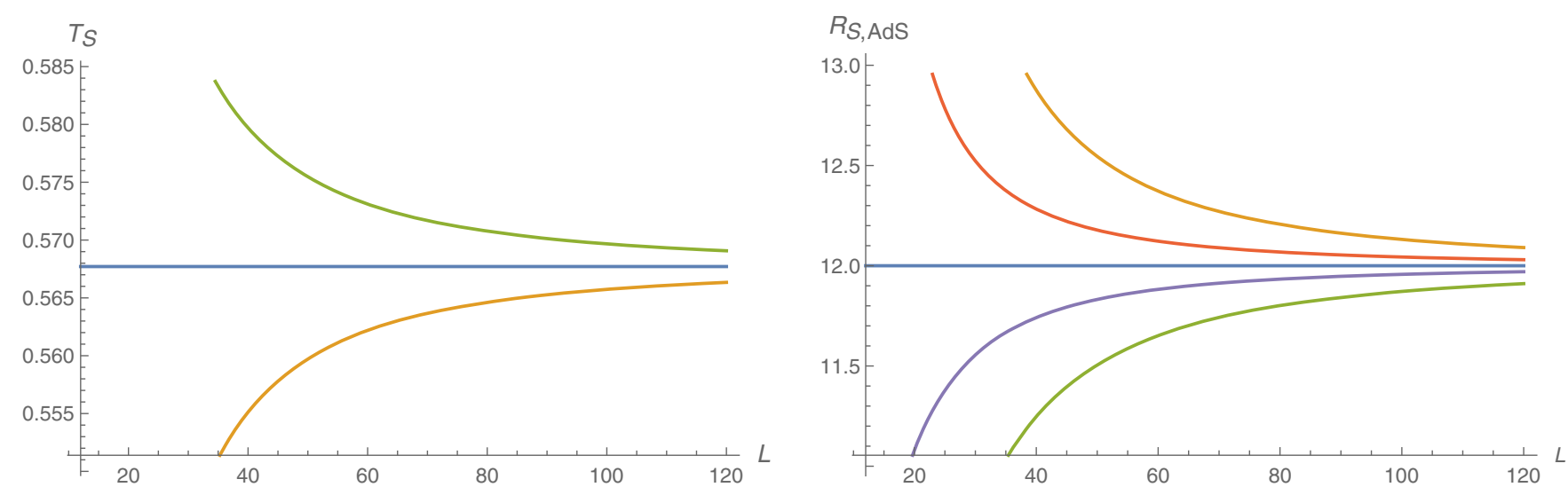

FIG. 1. (Left panel) The effective temperature $T_{\mathrm{S}}$ against $L$ with $Q=\sqrt{12}, q=\pi, m=1, l=0$ fixed. The green (upper) and yellow (lower) curves are the effective temperature for extremal RN black holes in AdS and dS spaces, respectively, while the blue horizontal line is that in asymptotically flat space. (Right panel) $\mathrm{AdS}_{2}$ radius $R_{\mathrm{AdS}}$ and the two-sphere radius $R_{\mathrm{S}}$ against $L$ with $Q=\sqrt{12}, q=\pi$, $m=1, l=0$ fixed. The horizontal line denotes both $R_{\text {AdS }}$ and $R_{\mathrm{S}}$ in the asymptotically flat space. The yellow (uppermost) curve denotes $R_{\mathrm{AdS}}$, and the orange curve above the horizontal line denotes $R_{\mathrm{S}}$ in dS space; the violet curve below the horizontal line denotes $R_{\mathrm{S}}$, and the green curve (lowermost) denotes $R_{\text {AdS }}$ in AdS space.

$$
\begin{aligned}
d s^{2} & =-\frac{\rho^{2}-B^{2}}{R_{\mathrm{AdS}}^{2}} d \tau^{2}+\frac{R_{\mathrm{AdS}}^{2}}{\rho^{2}-B^{2}} d \rho^{2}+R_{\mathrm{S}}^{2} d \Omega_{2}^{2}, \\
A & =-\frac{Q}{R_{\mathrm{S}}^{2}} \rho d \tau .
\end{aligned}
$$

The Hawking temperature and the chemical potential on the horizon in the metric (21) are then given by

$$
T_{\mathrm{H}}=\frac{B}{2 \pi R_{\mathrm{AdS}}^{2}}, \quad \Phi_{\mathrm{H}}=-\frac{Q B}{R_{\mathrm{S}}^{2}},
$$

while the Hawking temperature in the original metric is $\epsilon T_{\mathrm{H}}$ and suppressed by small $\epsilon$ due to the rescaling of time. The radial equation of the charged scalar field is

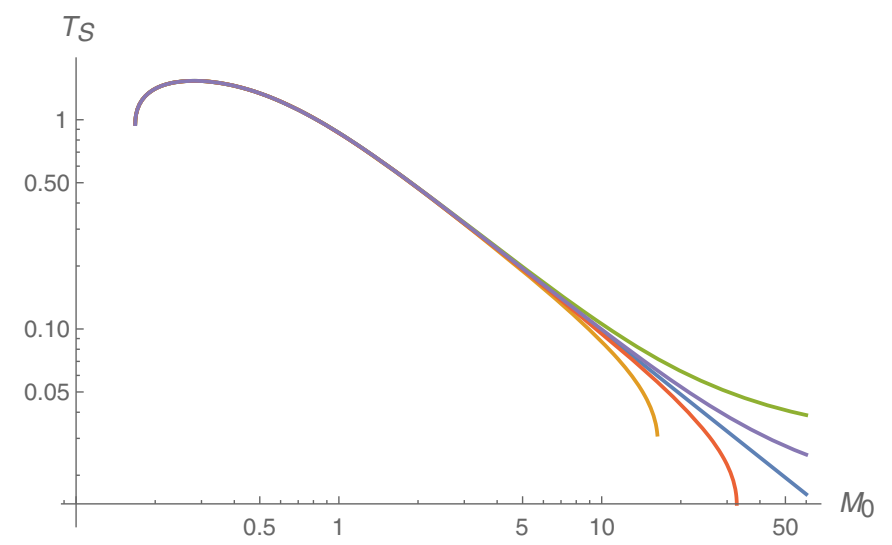

$$
\begin{aligned}
\partial_{\rho} & {\left[\left(\rho^{2}-B^{2}\right) \partial_{\rho} R\right]+\left[\frac{R_{\mathrm{AdS}}^{4}}{R_{\mathrm{S}}^{4}} \frac{\left(q Q \rho-\omega R_{\mathrm{S}}^{2}\right)^{2}}{\rho^{2}-B^{2}}-R_{\mathrm{AdS}}^{2} m^{2}\right.} \\
- & \left.\frac{R_{\mathrm{AdS}}^{2}}{R_{\mathrm{S}}^{2}} l(l+1)\right] R=0 .
\end{aligned}
$$

The solutions to the radial equation are found in terms of the hypergeometric functions, which leads to the mean number for pair production

$$
\begin{aligned}
\mathcal{N} & =\frac{\sinh (2 \pi \mu) \sinh (\pi \tilde{\kappa}-\pi \kappa)}{\cosh (\pi \kappa+\pi \mu) \cosh (\pi \tilde{\kappa}-\pi \mu)} \\
& =\frac{\mathrm{e}^{-2 \pi(\kappa-\mu)}-\mathrm{e}^{-2 \pi(\kappa+\mu)}}{1+\mathrm{e}^{-2 \pi(\kappa+\mu)}} \times \frac{1-\mathrm{e}^{-2 \pi(\tilde{\kappa}-\kappa)}}{1+\mathrm{e}^{-2 \pi(\tilde{\kappa}-\mu)}}
\end{aligned}
$$

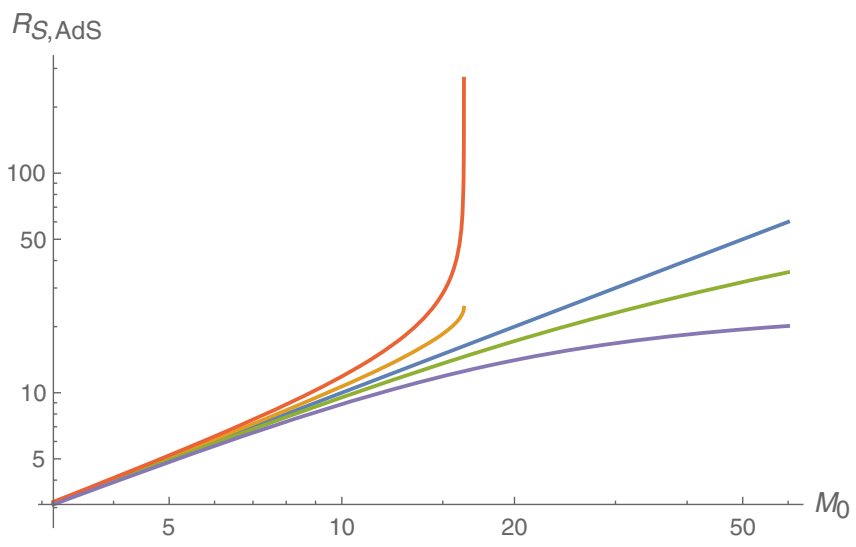

FIG. 2. (Left panel) The effective temperature $T_{\mathrm{S}}$ against $M_{0}$ with $q=\pi, m=1, l=0$ fixed. The blue (middle) curve is the effective temperature in the asymptotically flat space $(L=\infty)$. The green (uppermost) and purple (above the blue) curves are $T_{\mathrm{S}}$ with $L=60$ and $L=120$, respectively, in AdS space while the red (below the blue) and yellow (bottommost) curves are $T_{\mathrm{S}}$ with $L=120$ and $L=60$, respectively, in dS space. (Right panel) $\mathrm{AdS}_{2}$ radius $R_{\text {AdS }}$ and the two-sphere radius $R_{\mathrm{S}}$ against $M_{0}$ with $L=60, q=\pi, m=1, l=0$ fixed. The blue curve is $R_{\mathrm{AdS}}=R_{\mathrm{S}}$ in the asymptotically flat space. The red (uppermost) curve is $R_{\mathrm{AdS}}$, and the yellow (above the blue) curve is $R_{\mathrm{S}}$ in dS space; the green (below the blue) curve is $R_{\mathrm{S}}$, and the purple (bottommost) curve is $R_{\mathrm{AdS}}$ in AdS space. 
where

$$
\kappa=\frac{R_{\mathrm{AdS}}^{2}}{R_{\mathrm{S}}^{2}} q Q=-\frac{q \Phi_{\mathrm{H}}}{2 \pi T_{\mathrm{H}}}, \quad \tilde{\kappa}=\frac{\omega R_{\mathrm{AdS}}^{2}}{B}=\frac{\omega}{2 \pi T_{\mathrm{H}}} .
$$

The mean number has the following thermal interpretation [22]

$$
\begin{aligned}
\mathcal{N}= & \underbrace{\left(\frac{\mathrm{e}^{-\bar{m} / T_{\mathrm{S}}}-\mathrm{e}^{-\bar{m} / \bar{T}_{\mathrm{S}}}}{1+\mathrm{e}^{-\bar{m} / \bar{T}_{\mathrm{S}}}}\right)}_{\text {Schwinger effect in AdS }} \\
& \times\{\mathrm{e}^{\bar{m} / T_{\mathrm{S}}} \underbrace{}_{\text {Schwinger effect in Rindler }} \underbrace{\left(\mathrm{e}^{-\bar{m} / T_{\mathrm{S}}} \frac{1-\mathrm{e}^{-\left(\omega+q \Phi_{\mathrm{H}}\right) / T_{\mathrm{H}}}}{1+\mathrm{e}^{-\bar{m} / T_{\mathrm{S}}} \mathrm{e}^{-\left(\omega+q \Phi_{\mathrm{H}}\right) / T_{\mathrm{H}}}}\right)}\} .
\end{aligned}
$$

The first set of parentheses contains the Schwinger effect for an extremal black hole. The terms in curly brackets are expected since the Hawking temperature for a nearextremal black hole does not completely vanish, though small, and the Hawking radiation and the Schwinger effect are intertwined. The surface gravity of the event horizon determines the acceleration of the two-dimensional Rindler space, and the effective temperature is the QED effect in the electric field of a charged black hole.

\section{III. (NEAR-)EXTREMAL DYONIC RN-(A)dS BLACK HOLES}

The second model includes the dyonic RN-(A)dS black holes with the electric and magnetic charges $Q$ and $P$, with the metric and potentials

$$
\begin{aligned}
d s^{2} & =-f(r) d t^{2}+\frac{d r^{2}}{f(r)}+r^{2} d \Omega_{2}^{2}, \\
f(r) & =1-\frac{2 M}{r}+\frac{Q^{2}+P^{2}}{r^{2}} \pm \frac{r^{2}}{L^{2}}, \\
A & =\frac{Q}{r} d t+P(\cos \theta \mp 1) d \varphi, \\
\bar{A} & =\frac{P}{r} d t-Q(\cos \theta \mp 1) d \varphi,
\end{aligned}
$$

where the upper (lower) sign in $f(r)$ is for AdS (dS) space. The magnetic monopole induces a stringlike singularity causing different choices of the gauge potential: the upper sign is regular in $0 \leq \theta<\pi / 2$, and the lower sign is $\pi / 2<\theta \leq \pi$. The field strengths of $A$ and $\bar{A}$ are Hodge dual to each other. The extremal condition, $M_{0}$, and the radius of the degenerated horizon, $r_{0}$, are given in (3), with a modification of

$$
\delta= \pm\left(\sqrt{1 \pm 12\left(Q^{2}+P^{2}\right) / L^{2}}-1\right) .
$$

Note that parameters for extremal dyonic RN black holes are obtained by replacing $Q^{2}+P^{2}$ for $Q^{2}$ for extremal RN black holes. By stretching the radial coordinate $r=r_{0}+\epsilon \rho$ but squeezing the time coordinate $t=\tau / \epsilon$ and taking the near-extremal condition (20), we obtain the near-horizon geometry of near-extremal dyonic RN-(A)dS,

$$
\begin{aligned}
d s^{2} & =-\frac{\rho^{2}-B^{2}}{R_{\mathrm{AdS}}^{2}} d \tau^{2}+\frac{R_{\mathrm{AdS}}^{2}}{\rho^{2}-B^{2}} d \rho^{2}+R_{\mathrm{S}}^{2} d \Omega_{2}^{2}, \\
A & =-\frac{Q}{R_{\mathrm{S}}^{2}} \rho d \tau+P(\cos \theta \mp 1) d \varphi, \\
\bar{A} & =-\frac{P}{R_{\mathrm{S}}^{2}} \rho d \tau-Q(\cos \theta \mp 1) d \varphi,
\end{aligned}
$$

where the radii $R_{\mathrm{AdS}}$ and $R_{\mathrm{S}}$ are given in Eq. (5), with $\delta$ in Eq. (28). The electric and magnetic charges $q$ and $p$ of emitted particles are coupled to the potentials $A^{\mu}$ and $\bar{A}^{\mu}$ as

$\left(\nabla_{\mu}-i q A_{\mu}-i p \bar{A}_{\mu}\right)\left(\nabla^{\mu}-i q A^{\mu}-i p \bar{A}^{\mu}\right) \Phi-m^{2} \Phi=0$,

whose wave function takes the form

$$
\Phi(\tau, \rho, \theta, \varphi)=\mathrm{e}^{-i \omega \tau+i[n \mp(q P-p Q)] \varphi} R(\rho) S(\theta) .
$$

The solution separates into the angular and the radial parts

$$
\begin{gathered}
\frac{1}{\sin \theta} \partial_{\theta}\left(\sin \theta \partial_{\theta} S\right)-\left(\frac{[n-(q P-p Q) \cos \theta]^{2}}{\sin ^{2} \theta}-\lambda\right) S=0, \\
\partial_{\rho}\left[\left(\rho^{2}-B^{2}\right) \partial_{\rho} R\right]+\left[\frac{R_{\mathrm{AdS}}^{4}}{R_{\mathrm{S}}^{4}} \frac{\left[(q Q+p P) \rho-\omega R_{\mathrm{S}}^{2}\right]^{2}}{\rho^{2}-B^{2}}\right. \\
\left.-R_{\mathrm{AdS}}^{2} m^{2}-\frac{R_{\mathrm{AdS}}^{2}}{R_{\mathrm{S}}^{2}} \lambda\right] R=0 .
\end{gathered}
$$

The result is analogous to an electric black hole with the modifications

$\kappa=\frac{R_{\mathrm{AdS}}^{2}}{R_{\mathrm{S}}^{2}}(q Q+p P)=-\frac{q \Phi_{\mathrm{H}}+p \bar{\Phi}_{\mathrm{H}}}{2 \pi T_{\mathrm{H}}}, \quad \bar{\Phi}_{\mathrm{H}}=-\frac{P B}{R_{\mathrm{S}}^{2}}$.

The mean number generalizes the formula with zero angular momentum in Ref. [7] to the (A)dS boundary 


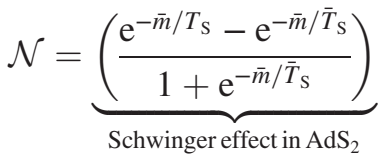

$$
\begin{aligned}
& \times\{\mathrm{e}^{\bar{m} / T_{\mathrm{S}}} \underbrace{\left(\mathrm{e}^{-\bar{m} / T_{\mathrm{S}}} \frac{1-\mathrm{e}^{-\left(\omega+q \Phi_{\mathrm{H}}+p \bar{\Phi}_{\mathrm{H}}\right) / T_{\mathrm{H}}}}{1+\mathrm{e}^{-\bar{m} / T_{\mathrm{S}}} \mathrm{e}^{-\left(\omega+q \Phi_{\mathrm{H}}+p \bar{\Phi}_{\mathrm{H}}\right) / T_{\mathrm{H}}}}\right)}_{\text {Schwinger effect in Rindler } 2}\} .
\end{aligned}
$$

The Schwinger temperatures, $T_{\mathrm{S}}$ and $\bar{T}_{\mathrm{S}}$, are given in Eq. (19) with a generalized $\kappa$ in Eq. (34). It should be noted that the mean number (35) for Schwinger pair production has the universal form as Eq. (26) for near-extremal RN black holes: the factorization of the Schwinger effect into an $\mathrm{AdS}_{2}$ and a two-dimensional Rindler space. The charge emission via Hawking radiation is given by the Hawking temperature with chemical potentials for electric and magnetic charges, which is intertangled with the Schwinger term.

\section{CONCLUSION}

We have studied the Schwinger effect from (near-) extremal RN black holes with electric and/or magnetic charges in (A)dS space. It is found that the asymptotic (A) dS boundary drastically changes the $\mathrm{AdS}_{2}$ and $S^{2}$ radii of near-horizon geometry and the effective temperature for the
Schwinger effect, and this boundary effect increases the Schwinger pair production for AdS space but decreases it for dS space. The smaller the radius $L$ of AdS/dS space is, the larger the enhancement/suppression of the Schwinger effect is. A physical reasoning for this phenomenon is that the AdS asymptotic boundary pushes the event horizon inward and strengthens the electric field on it, while the dS boundary pulls the event horizon toward the cosmological horizon and weakens the field on the event horizon. One interesting cosmological implication is that dyonic (near-) extremal RN or KN black holes are larger and have longer lifetimes in dS space than those in asymptotically flat space; these primordial black holes may be candidates for dark matter (for constraints for primordial black holes, see Ref. [23]), and their binaries may provide a source for gravitational waves [24]. This issue and other cosmological and astrophysical implications will be addressed in a separate paper.

\section{ACKNOWLEDGMENTS}

S.P.K. would like to thank the National Central University for warm hospitality. The work of C. M. C. was supported by the Ministry of Science and Technology of the R.O.C. under Grant No. MOST 108-2112-M-008007. The work of S.P. K. was supported in part by the National Research Foundation of Korea (NRF) funded by the Ministry of Education (2019R1I1A3A01063183).
[1] S. W. Hawking, Particle creation by black holes, Commun. Math. Phys. 43, 199 (1975); Erratum, Commun. Math. Phys. 46, 206 (1976).

[2] F. Sauter, Zum Kleinschen paradoxon, Z. Phys. 73, 547 (1932).

[3] J.S. Schwinger, On gauge invariance and vacuum polarization, Phys. Rev. 82, 664 (1951).

[4] C.-M. Chen, S. P. Kim, I.-C. Lin, J.-R. Sun, and M.-F. Wu, Spontaneous pair production in Reissner-Nordstrom black holes, Phys. Rev. D 85, 124041 (2012).

[5] C.-M. Chen, J.-R. Sun, F.-Y. Tang, and P.-Y. Tsai, Spinor particle creation in near extremal ReissnerNordstrom black holes, Classical Quantum Gravity 32, 195003 (2015).

[6] C.-M. Chen, S. P. Kim, J.-R. Sun, and F.-Y. Tang, Pair production in near extremal Kerr-Newman black holes, Phys. Rev. D 95, 044043 (2017).

[7] C.-M. Chen, S. P. Kim, J.-R. Sun, and F.-Y. Tang, Pair production of scalar dyons in Kerr-Newman black holes, Phys. Lett. B 781, 129 (2018).

[8] S. P. Kim, Astrophysics in strong electromagnetic fields and laboratory astrophysics, arXiv:1905.13439.

[9] F. Belgiorno and S. L. Cacciatori, Quantum effects for the Dirac field in Reissner-Nordstrom-AdS black hole background, Classical Quantum Gravity 25, 105013 (2008).

[10] F. Belgiorno and S. L. Cacciatori, Massive Dirac particles on the background of charged de-Sitter black hole manifolds, Phys. Rev. D 79, 124024 (2009).

[11] F. Belgiorno, S. L. Cacciatori, and F. Dalla Piazza, Quantum instability for charged scalar particles on charged Nariai and ultracold black hole manifolds, Classical Quantum Gravity 27, 055011 (2010).

[12] S. Hawking, Gravitationally collapsed objects of very low mass, Mon. Not. R. Astron. Soc. 152, 75 (1971).

[13] B. J. Carr and S. W. Hawking, Black holes in the early Universe, Mon. Not. R. Astron. Soc. 168, 399 (1974).

[14] B.J. Carr, The primordial black hole mass spectrum, Astrophys. J. 201, 1 (1975).

[15] G. W. Gibbons, Black hole dyons need not explode, Phys. Rev. D 15, 3530 (1977).

[16] Y. Aharonov, A. Casher, and S. Nussinov, The unitarity puzzle and Planck mass stable particles, Phys. Lett. B 191, 51 (1987).

[17] M. Montero, T. Van Riet, and G. Venken, Festina Lente: EFT constraints from charged black hole evaporation in de Sitter, J. High Energy Phys. 01 (2020) 039. 
[18] L. J. Romans, Supersymmetric, cold and lukewarm black holes in cosmological Einstein-Maxwell theory, Nucl. Phys. B383, 395 (1992).

[19] B. Pioline and J. Troost, Schwinger pair production in AdS (2), J. High Energy Phys. 03 (2005) 043.

[20] S. P. Kim and D. N. Page, Schwinger pair production in dS(2) and AdS(2), Phys. Rev. D 78, 103517 (2008).

[21] R.-G. Cai and S. P. Kim, One-loop effective action and Schwinger effect in (anti-)de Sitter space, J. High Energy Phys. 09 (2014) 072.
[22] S. P. Kim, H. K. Lee, and Y. Yoon, Thermal interpretation of Schwinger effect in near-extremal RN black hole, Int. J. Mod. Phys. D 28, 1950139 (2019).

[23] B. J. Carr, K. Kohri, Y. Sendouda, and J. Yokoyama, New cosmological constraints on primordial black holes, Phys. Rev. D 81, 104019 (2010).

[24] L. Liu, Z.-K. Guo, R.-G. Cai, and S. P. Kim, Merger rate distribution of primordial black hole binaries with electric charges, arXiv:2001.02984. 